\title{
fMRI changes over time and reproducibility in unmedicated subjects at high genetic risk of schizophrenia
}

\author{
H. C. Whalley*, V.-E. Gountouna, J. Hall, A. M. McIntosh, E. Simonotto, D. E. Job, D. G. C. Owens, \\ E. C. Johnstone and S. M. Lawrie
}

Division of Psychiatry, School of Molecular and Clinical Medicine, University of Edinburgh, Edinburgh, UK

\begin{abstract}
Background. Functional brain abnormalities have been repeatedly demonstrated in schizophrenia but there is little data concerning their progression. For such studies to have credibility it is first important to establish the reproducibility of functional imaging techniques. The current study aimed to examine these factors in healthy controls and in unmedicated subjects at high genetic risk of the disorder: (i) to examine the reproducibility of taskrelated activation patterns, (ii) to determine if there were any progressive functional changes in high-risk subjects versus controls reflecting inheritance of the schizophrenic trait, and (iii) to examine changes over time in relation to fluctuating positive psychotic symptoms (i.e. state effects).
\end{abstract}

Method. Subjects were scanned performing the Hayling sentence completion test on two occasions 18 months apart. Changes in activation were examined in controls and high-risk subjects $(n=16, n=63)$. Reproducibility was assessed for controls and high-risk subjects who remained asymptomatic at both time points $(n=16, n=32)$.

Results. Intra-class correlation values indicated good agreement between scanning sessions. No significant differences over time were seen between the high-risk and control group; however, comparison of high-risk subjects who developed symptoms versus those who remained asymptomatic revealed activation increases in the left middle temporal gyrus $(p=0.026)$.

Conclusions. The current results suggest that functional changes over time occur in the lateral temporal cortex as high genetic risk subjects become symptomatic, further, they indicate the usefulness of functional imaging tools for investigating progressive changes associated with state and trait effects in schizophrenia.

Received 27 February 2008; Revised 21 October 2008; Accepted 5 November 2008; First published online 24 December 2008

Key words: fMRI, longitudinal, schizophrenia.

\section{Introduction}

Although it is well established that patients with schizophrenia have demonstrable structural and functional brain abnormalities in prefrontal and temporal regions, much less is known about brain changes over time in association with the disorder (Shenton et al. 2001; Fusar-Poli et al. 2007). This issue has central importance with regard to understanding the nature of the disease, in terms of whether abnormalities are neurodevelopmental in origin, whether they are progressive, or a combination of the two. Indeed, the lack of unequivocal evidence for clinical or biological progression in the established condition could infer that the period of greatest change occurs prior to development of the disorder and could relate to inheritance of

\footnotetext{
* Address for correspondence: H. C. Whalley, Ph.D., Division of Psychiatry, University of Edinburgh, Kennedy Tower, Royal Edinburgh Hospital, Morningside Park, Edinburgh EH10 5HF, UK. (Email: hwhalley@staffmail.ed.ac.uk)
}

the schizophrenic trait, or alternatively may reflect subtle changes in association with evolving symptomatology. Hence, there is interest in studying the relationship between functional brain abnormalities and the course and early symptoms of the disorder.

There is a paucity of data tracking the progression of functional abnormalities in high-risk populations. Longitudinal functional magnetic resonance imaging (fMRI) studies have, however, previously been conducted in patients with established schizophrenia and other clinical populations as well as in healthy control subjects, indicating the feasibility of the technique to examine changes in brain function (Ward et al. 2003; Tombari et al. 2004; Yoo et al. 2005; Szaflarski et al. 2006; Marchand et al. 2007). There is also a growing body of literature directly addressing issues relating to the reliability of fMRI responses, which has obvious importance for longitudinal studies. In general such studies have suggested that, in healthy controls at least, although there may be variability in the volume 
of response, there are consistent patterns of activation between scanning sessions at the group level (Casey et al. 1998; Machielsen et al. 2000; Chee et al. 2003). Functional imaging techniques are therefore of interest in the investigation of longitudinal changes related to schizophrenia in those at high risk of developing the disorder. It should be noted, however, that there are as yet relatively few reproducibility studies in patient populations (Manoach et al. 2001; Chen \& Small, 2007) and it is therefore important that reliability of $\mathrm{fMRI}$ in such populations is addresed.

The current study examines subjects from the Edinburgh High Risk Study (EHRS). This was a prospective 10-year longitudinal study designed to address issues relating to genetic vulnerability to psychosis (Johnstone et al. 2002, 2005). Functional imaging was introduced in the last 5 years of the study and involved a sentence completion paradigm known to activate regions reported to be abnormal in established schizophrenia (frontal and temporal lobes). The aim of the current study was to address three issues: (i) the reliability of the Hayling task in high-risk and control subjects; (ii) whether longitudinal changes occur in high-risk subjects who differ from healthy control subjects reflecting inheritance of the schizophrenic 'trait'; (iii) whether longitudinal changes occur in high-risk subjects relating to fluctuations in psychotic symptoms, reflecting 'state'-related effects. Since the study involved high-risk participants who did not meet diagnostic criteria for a psychiatric disorder, the term 'state' here is used to describe partial phenotypic expression of some of the characteristic symptoms of schizophrenia, rather than full phenotypic expression of the disease.

Based on previous cross-sectional functional imaging studies relating to positive psychotic symptoms (Cleghorn et al. 1992; Suzuki et al. 1993; David et al. 1996; Dierks et al. 1999; Lennox et al. 2000; Shergill et al. 2000 ; Stephane et al. 2000; van de Ven et al. 2005), and longitudinal structural imaging studies regarding the importance of temporal lobe changes in early schizophrenia (Kasai et al. 2003a,b; Whitford et al. 2006) and high-risk subjects (Thompson et al. 2001; Lawrie et al. 2002; Pantelis et al. 2003; Job et al. 2005), and in our own cross-sectional fMRI study in highrisk subjects (Whalley et al. 2007), we hypothesized changes in temporal lobe activation in high-risk subjects relating to changes in symptomatic state.

\section{Method}

\section{Subject details}

The EHRS examines young adults at enhanced genetic risk of schizophrenia over the period at which they are at greatest risk of becoming ill (Hafner et al. 1993) in comparison with matched healthy controls (Johnstone et al. 2002). The high-risk and control subjects were matched as closely as possible at recruitment for age, gender and social class at birth based on father's occupation (see Byrne et al. 1999). High-risk participants were selected on the basis of being aged between 16 and 25 years when first recruited (1994-1999), and having one first- or second-degree relative with schizophrenia and a minimum of one further genetic relative with the illness (Hodges et al. 1999; Johnstone et al. 2002, 2005). This report concerns longitudinal fMRI data obtained during the second phase of the study (1999-2004) when functional imaging was introduced into the protocol. All subjects were supplied with detailed written information regarding the study and provided written informed consent. The study was approved by the Psychiatry and Clinical Psychology subcommittee of the Lothian research ethics committee.

During the study, a total of 96 high-risk subjects and 26 matched healthy controls underwent an fMRI scan at baseline. Sixty-three high-risk subjects and 19 control participants provided a repeat scan after approximately 18 months. Regarding the high-risk group, 33 subjects (comprising 19 asymptomatic and 14 symptomatic subjects at baseline) did not have a repeat scan: 10 refused or had withdrawn, seven were excluded because of abnormality or movement artefact, two had become ill between assessments, for six subjects it was not possible to schedule a suitable time for assessment, and finally due to rolling recruitment a suitable time interval between assessments had not elapsed in eight subjects. For the controls, out of the seven subjects who did not have a repeat scan, two refused or had withdrawn, and three subjects were not re-scanned due to rolling recruitment. The remainder had either previously accompanied a participant who had subsequently refused or withdrawn from the study, or it was not possible to schedule a suitable time for assessment.

Participant demographics are presented in Table 1. At the time of scanning all subjects underwent a structured psychiatric interview, the Present State Examination (PSE; Wing et al. 1974). These interviews, conducted by experienced psychiatrists (E.C.J. and D.G.C.O.), were filmed, and videotapes have been retained. The PSE was chosen as the primary method to determine the presence or absence of psychotic symptoms in this study as it is a detailed instrument providing a standardized assessment of a wide range of symptomatology, which we considered useful in the context of establishing the extent of psychopathology in the high-risk and control subjects (Johnstone et al. 2000). Subjects were then split according to the 
Table 1. Demographics

\begin{tabular}{llllll}
\hline & $\begin{array}{l}\mathrm{C}_{\mathrm{NN}} \\
(n=16)\end{array}$ & $\begin{array}{l}\mathrm{HR}_{\mathrm{NN}} \\
(n=32)\end{array}$ & $\begin{array}{l}\mathrm{HR}_{\mathrm{NP}} \\
(n=9)\end{array}$ & $\begin{array}{l}\mathrm{HR}_{\mathrm{PN}} \\
(n=15)\end{array}$ & $\begin{array}{l}\mathrm{HR}_{\mathrm{PP}} \\
(n=5)\end{array}$ \\
\hline $\begin{array}{l}\text { Mean age at first functional } \\
\text { scan, years (s.D.) }\end{array}$ & $26.1(2.2)$ & $27.5(2.4)$ & $25.0(2.6)$ & $25.4(3.3)$ & $27.3(2.8)$ \\
$\begin{array}{l}\text { Gender, } n \\
\quad \text { Male }\end{array}$ & 10 & 17 & 3 & 6 & 2 \\
$\quad$ Female & 5 & 15 & 6 & 9 & 3 \\
$\begin{array}{l}\text { Time interval, years } \\
\quad \text { Mean (s.D.) }\end{array}$ & $1.6(0.6)$ & $1.5(0.6)$ & $1.6(0.3)$ & $1.3(0.3)$ & $1.3(0.3)$ \\
$\quad$ Range & $1.0-3.7$ & $0.9-3.4$ & $0.9-2.0$ & $0.7-1.8$ & $0.9-1.6$ \\
\hline
\end{tabular}

$\mathrm{C}_{\mathrm{NN}}$, Control subjects with no psychotic symptoms at times 1 and $2 ; \mathrm{HR}_{\mathrm{NN}}$, high-risk subjects with no psychotic symptoms at times 1 and $2 ; \mathrm{HR}_{\mathrm{NP}}$, high-risk subjects with no psychotic symptoms at time 1 but with psychotic symptoms at time $2 ; \mathrm{HR}_{\mathrm{PN}}$, high-risk subjects with psychotic symptoms at time 1 but with none at time $2 ; \mathrm{HR}_{\mathrm{PP}}$, high-risk subjects with psychotic symptoms at both time 1 and time 2 .

presence or absence of psychotic symptoms. Our notation of symptomatic or asymptomatic participants refers specifically to the presence of isolated and/or transient psychotic symptoms reported at interview. Thirty-two high-risk subjects were found to be asymptomatic at both time points $\left(\mathrm{HR}_{\mathrm{NN}}\right)$, nine subjects went from asymptomatic to symptomatic $\left(\mathrm{HR}_{\mathrm{NP}}\right)$, fifteen went from symptomatic to asymptomatic $\left(\mathrm{HR}_{\mathrm{PN}}\right)$, five subjects were symptomatic at both time points $\left(\mathrm{HR}_{\mathrm{PP}}\right)$, and finally two subjects went from being symptomatic to developing schizophrenia. The diagnosis was made when psychotic symptoms were sufficiently severe or sustained to satisfy categorization in terms of PSE/Catego (Wing et al. 1974) and ICD-10 (WHO, 1993) criteria as previously described (Johnstone et al. 2005). Due to this small number it was not possible to examine progressive changes preceding illness. Regarding the control subjects, sixteen subjects were asymptomatic at both time points $\left(\mathrm{C}_{\mathrm{NN}}\right)$, and three subjects went from asymptomatic to symptomatic. Analysis of demographic variables and behavioural measures was conducted in the Statistical Package for the Social Sciences (SPSS version 14; SPSS Inc., Chicago, IL, USA). There were no significant differences in age, gender, handedness or symptomatic status between those who were rescanned and those that were not. Due to small numbers of subjects in the groups containing high-risk subjects who went from being symptomatic to ill $(n=2)$, and control subjects who went from being asymptomatic to symptomatic $(n=3)$, subsequent analyses were conducted excluding these groups.

For the purposes of the trait contrast, group comparisons were conducted between the control and high-risk groups. For the purposes of the state contrast, group comparisons were conducted between the $\mathrm{HR}_{\mathrm{NN}}$ and the $\mathrm{HR}_{\mathrm{NP}}$ groups and between the $\mathrm{HR}_{\mathrm{NN}}$ and the $\mathrm{HR}_{\mathrm{PN}}$ groups.

\section{Scanning procedure}

Imaging was carried out at the Brain Imaging Research Centre (BIRC) for Scotland on a GE 1.5 T Signa scanner (GE Medical, Milwaukee, WI, USA). The imaging protocol consisted of a localizer scan, followed by a T2-weighted fast spin-echo sequence, and a structural T1-weighted sequence followed by the functional imaging paradigms. Axial gradient-echo planar images (EPI) [repetition time $4000 \mathrm{~ms}$, echo time $40 \mathrm{~ms}$; matrix $=64 \times 128$; field of view (FOV) $220 \times 440 \mathrm{~mm}$ ] were acquired continually. Thirty-eight contiguous $5 \mathrm{~mm}$ slices aligned to the anterior and posterior commissure were acquired within each repetition time period. Each acquisition was run for 204 volumes, of which the first four volumes were discarded.

\section{Experimental paradigm}

Participants performed the verbal initiation section of the Hayling sentence completion test in the scanner as described previously (Burgess \& Shallice, 1997; Whalley et al. 2004). Subjects were shown sentences with the last word missing and were asked to silently think of an appropriate word to complete the sentence and press a button when they had done so, generating within-scanner reaction time measures. Sentences were presented in blocks of fixed difficulty, determined by the range of suitable words suggested by the sentence context, of which there were four levels. Each block lasted $40 \mathrm{~s}$ and included eight sentences. Sentences were presented for a period of $3 \mathrm{~s}$ followed 
by a fixation cross for $2 \mathrm{~s}$ and subjects could respond at any time until the next sentence appeared. The baseline condition consisted of viewing a screen of white circles on a black background for $40 \mathrm{~s}$. The order of the blocks was pseudo-random, and each block was repeated four times. Immediately after scanning, subjects were given the same sequence of sentences on paper and requested to complete each sentence with the word they first thought of in the scanner. 'Word appropriateness' scores were determined from the word frequency list of sentence completion norms (Bloom \& Fischler, 1980). For more detail, see Whalley et al. (2004).

\section{Image processing and analysis}

Scan analysis was performed using the standard Statistical Parametric Mapping (SPM) approach in SPM2 (The Wellcome Department of Cognitive Neurology and collaborators, Institute of Neurology, London; http://www.fil.ion.ucl.ac.uk/spm/) running in MATLAB (MathWorks, Natick, MA, USA). Briefly, for both scanning sessions EPI volumes were realigned to the mean volume in the series, normalized to a studyspecific EPI template (comprising 121 participants of the EHRS) and spatially smoothed with a $6 \times 6 \times 6 \mathrm{~mm}^{3}$ full-width half-maximum (FWHM) Gaussian filter.

\section{First-level analysis}

Statistical analysis was performed using the general linear model approach as implemented in SPM. At the individual subject level the data were modelled with five conditions (the four difficulty levels and baseline), each modelled by a boxcar function convolved with a synthetic haemodynamic response function. Estimates of head movement from the realignment stage of preprocessing were included as additional regressors in the analysis. Before fitting the model the first-order autoregressive (AR-1) technique was used to address issues of temporal autocorrelations in the data and the data were filtered in the time domain using a highpass filter (200 s cut-off). Contrasts were constructed to examine all four sentence completion conditions versus baseline, and a parametric contrast which examines increasing activation with increasing difficulty (Whalley et al. 2004).

\section{Second-level analysis}

Contrast images for each subject representing a subject-specific summary of brain responses to the different conditions were then entered into secondlevel random-effects analyses. Initially, a singlesample $t$ test was used to generate within-group maps for each time point for both sentence completion versus baseline and the parametric contrast. Within-subject 'change' maps were then generated for sentence completion versus baseline and the parametric contrast by subtracting for each subject the respective contrast images from visit 1 from visit 2, divided by the time interval between the scanning sessions. Although there were no significant group differences in the time interval between the scans, the time interval was included to control for within-subject variability related to the between-scan interval. The individual subject change maps were then entered into further randomeffects analyses to examine changes in activation within (one-sample $t$ test) and between (analysis of variance; ANOVA) each of the groups. To test the concurrent validity of this approach, results from the active versus baseline contrast were compared with a more complex repeated-measures analysis of covariance (ANCOVA) model. Here, contrast images for each individual from time 1 and time 2 were entered as separate regressors into the model (per group) and the time interval between the scans was entered as a covariate of no interest. Contrasts were then constructed to examine activation differences between the groups. The two approaches yielded a similar pattern of results; therefore, the simpler 'one contrast image per subject' subtraction model was used.

Statistical maps were thresholded at a level of $p=0.001$ uncorrected, and regions were considered significant at $p<0.05$ cluster level-corrected for multiple comparisons. All $p$ values quoted in the text are at the corrected cluster level. A small volume correction for the temporal lobes was applied to contrasts examining state effects in line with our a priori hypothesis of change in activation in this region with symptom development.

\section{Reliability measures}

Reliability was assessed using approaches similar to those described previously (Specht et al. 2003; Aron et al. 2006). Measures were computed for the $C_{N N}$ and $\mathrm{HR}_{\mathrm{NN}}$ groups only in order that reliability measures were not confounded by symptom effects seen in the other groups. Intra-class correlation coefficient (ICC) values were calculated on a voxel-by-voxel basis using a one-way ANOVA model (Specht et al. 2003) on contrast images representing sentence completion versus baseline. High ICC values suggest low within-subject variability. The resulting three-dimensional ICC maps of ICC values $>0.5$ were then masked with a binary image representing the main network of regions involved in the task at visit 1 or visit 2 (Aron et al. 2006). The mask was generated by performing a one-sample $t$ test on subjects at visit 1 and at visit 2 . The resulting group activation maps for visit 1 and visit 2 were then thresholded at $p=0.01$ [ $p$ corrected family-wise error 
Table 2. Behavioural measures ${ }^{\mathrm{a}}$

\begin{tabular}{|c|c|c|c|c|c|}
\hline & $\mathrm{C}_{\mathrm{NN}}$ & $\mathrm{HR}_{\mathrm{NN}}$ & $\mathrm{HR}_{\mathrm{NP}}$ & $\mathrm{HR}_{\mathrm{PN}}$ & $\mathrm{HR}_{\mathrm{PP}}$ \\
\hline \multicolumn{6}{|c|}{ Word appropriateness score visit 1} \\
\hline Low & $6.3(1.4)$ & $6.4(0.8)$ & $5.9(1.3)$ & $6.4(1.0)$ & $6.4(0.6)$ \\
\hline Medium low & $3.1(0.5)$ & $3.2(0.6)$ & $3.3(0.5)$ & $3.4(0.6)$ & $3.3(0.5)$ \\
\hline Medium high & $1.8(0.3)$ & $2.0(0.4)$ & $1.8(0.3)$ & $2.0(0.4)$ & $2.0(0.3)$ \\
\hline High & $1.1(0.7)$ & $1.1(0.1)$ & $1.1(0.1)$ & $1.1(0.1)$ & $1.1(0.1)$ \\
\hline \multicolumn{6}{|c|}{ Word appropriateness score visit 2} \\
\hline Low & $6.0(1.0)$ & $6.0(1.0)$ & $6.5(1.0)$ & $6.2(1.0)$ & $6.7(0.5)$ \\
\hline Medium low & $3.0(0.4)$ & $3.1(0.6)$ & $3.0(0.5)$ & $3.2(0.6)$ & $3.1(0.4)$ \\
\hline Medium high & $1.9(0.3)$ & $1.8(0.3)$ & $1.8(0.3)$ & $1.9(0.3)$ & $2.0(0.2)$ \\
\hline High & $1.1(0.1)$ & $1.1(0.1)$ & $1.1(0.1)$ & $1.1(0.1)$ & $1.1(0.1)$ \\
\hline \multicolumn{6}{|c|}{ Reaction time visit $1, \mathrm{~ms}$} \\
\hline Low & $2613(440)$ & $2586(581)$ & $2163(433)$ & $2723(795)$ & $2635(597)$ \\
\hline Medium low & $2479(540)$ & $2488(642)$ & $2022(474)$ & $2652(811)$ & $2490(599)$ \\
\hline Medium high & $2357(560)$ & $2360(655)$ & $1847(389)$ & $2491(837)$ & $2469(745)$ \\
\hline High & $2291(589)$ & $2357(666)$ & $1846(446)$ & $2493(820)$ & $2373(667)$ \\
\hline \multicolumn{6}{|c|}{ Reaction time visit $2, \mathrm{~ms}$} \\
\hline Low & $2681(684)$ & $2505(603)$ & $2060(502)$ & $2829(706)$ & $2323(363)$ \\
\hline Medium low & $2610(735)$ & $2449(615)$ & $2051(437)$ & $2673(406)$ & $2244(378)$ \\
\hline Medium high & $2535(770)$ & $2306(630)$ & $1941(450)$ & $2574(746)$ & $2088(419)$ \\
\hline High & $2448(758)$ & $2310(593)$ & $1919(430)$ & $2565(718)$ & $2119(448)$ \\
\hline
\end{tabular}

$\mathrm{C}_{\mathrm{NN}}$, Control subjects with no psychotic symptoms at times 1 and $2 ; \mathrm{HR}_{\mathrm{NN}}$, high-risk subjects with no psychotic symptoms at times 1 and $2 ; \mathrm{HR}_{\mathrm{NP}}$, high-risk subjects with no psychotic symptoms at time 1 but with psychotic symptoms at time 2 ; $\mathrm{HR}_{\mathrm{PN}}$, high-risk subjects with psychotic symptoms at time 1 but with none at time $2 ; \mathrm{HR}_{\mathrm{PP}}$, high-risk subjects with psychotic symptoms at both time 1 and time 2 .

Values are given as mean (standard deviation).

${ }^{a}$ Constraint levels high to low represent increasing task difficulty. Low word appropriateness scores represent more appropriate words, i.e. better task performance. Reaction times were recorded in the scanner.

(FWE), cluster extent threshold in voxels $\left.\left(\mathrm{K}_{\mathrm{E}}\right)=500\right]$, and a binary mask created. ICC maps therefore only present voxels that were activated at visit 1 or at visit 2 .

The mean response for three main regions of interest (left superior/medial frontal gyrus, left middle temporal gyrus and left inferior frontal gyrus) for each subject and each visit was also extracted in order to generate signal plots of visit 1 versus visit 2 . The coordinates for the three main regions of interest were identified from the cluster results from an all-subjects second-level random-effects analysis; the left superior/middle frontal gyrus $(-4,4,56)$, the left middle temporal gyrus $(-54,-38,-6)$ and the left inferior frontal gyrus $(-54,14,14)$ and values were extracted for a $10 \mathrm{~mm}$ radius sphere centred on these coordinates.

\section{Results}

\section{Subject groups}

There were no statistically significant differences between the groups regarding the time interval between the scans, or gender. There was, however, a significant difference in age at the time of the first functional scan between the groups $(p=0.049)$. The results presented below survived controlling for age. Behavioural measures for each time point are presented in Table 2 . These indicated the expected pattern of quicker reaction time and lower word appropriateness scores with greater contextual constraint at both assessments and indicate that the subjects were performing the task appropriately in the scanner. There were no significant group $\times$ time interactions for any of the behavioural measures (general linear model repeated-measures ANCOVA).

\section{Reliability measures}

Fig. 1 presents within-group maps at each time point for sentence completion versus baseline, providing a means of qualitative assessment of reliability. Fig. 1 shows that there were consistent activation patterns across time in both groups in regions previously associated with the task, namely the left precentral gyrus, inferior frontal gyrus, medial/superior frontal 
(a)

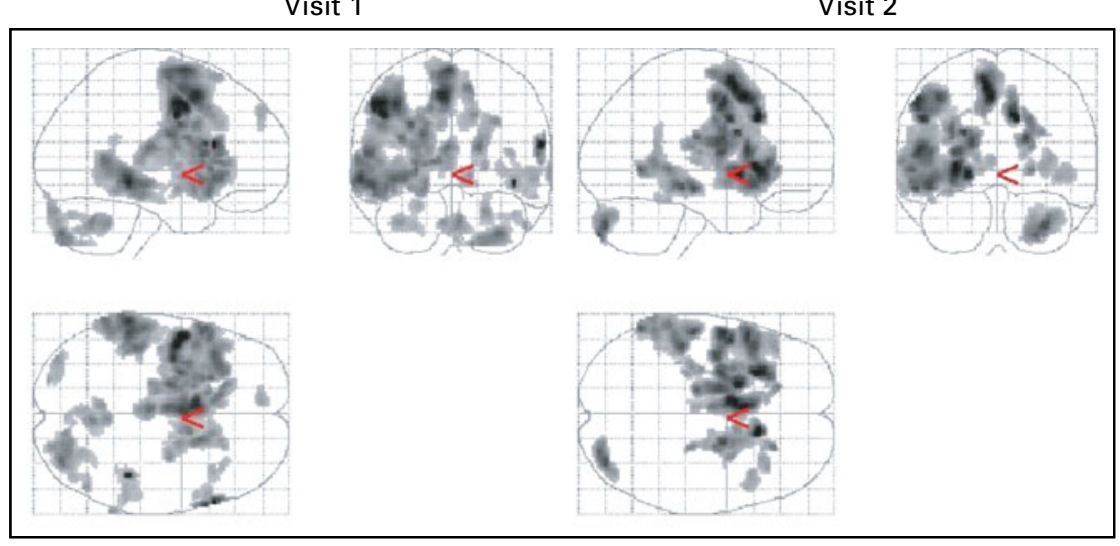

(b)

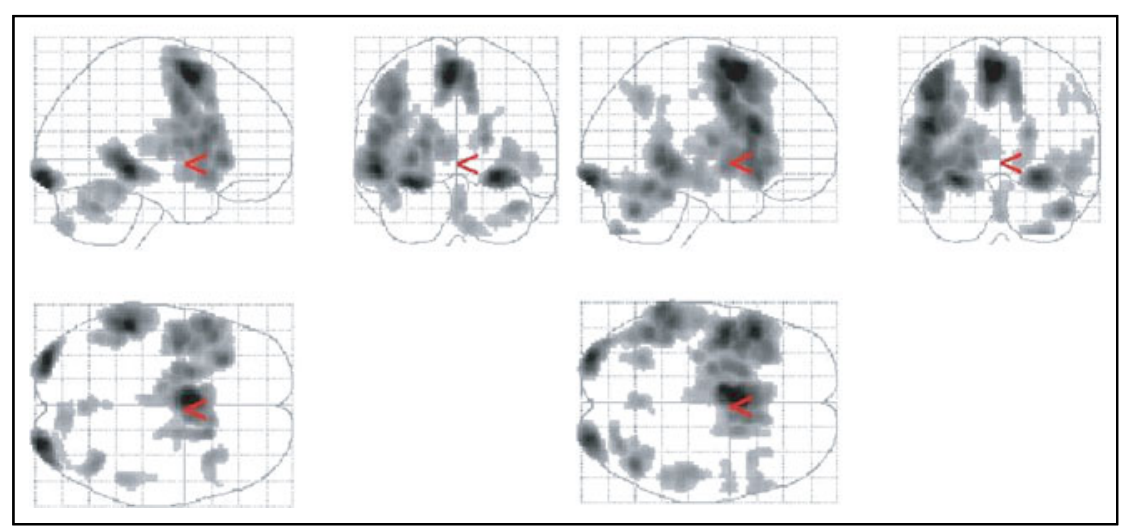

Fig. 1. Sentence completion versus baseline maps (a) for control subjects $(n=16)$ at visits 1 and 2, thresholded at $\mathrm{T}=3.5$, cluster extent threshold in voxels $\left(\mathrm{K}_{\mathrm{E}}\right)=100$, and $(b)$ maps for high-risk subjects $(n=61)$ for visits 1 and 2 , thresholded at $\mathrm{T}=5.5$, $\mathrm{K}_{\mathrm{E}}=100$. Maps were thresholded at different levels for visualization purposes only in order to accommodate differences in group numbers. Standard statistical thresholds were used in all group comparisons.

gyrus, middle temporal gyrus, and the right posterior lobe of the cerebellum.

ICC maps for the main regions activated for the sentence completion versus baseline contrast are presented in Fig. 2(a,b) for both groups for voxels activated at either visit 1 or visit 2 . Plots of the mean response in these regions for visits 1 and 2 are presented in Fig. $2 c$ and indicate that responses were highly correlated and were similar across the groups. ICC values for the mean responses for the three main regions of interest are presented in Table 3. Values ranged from 0.55 to 0.65 for the controls and from 0.65 to 0.80 for the $\mathrm{HR}_{\mathrm{NN}}$ group, indicating good agreement.

\section{Sentence completion versus baseline contrast}

\section{Trait effects}

Formal statistical comparisons revealed no significant differences between the groups at the chosen levels of significance.

\section{State effects}

Comparison of the $\mathrm{HR}_{\mathrm{NN}}$ and $\mathrm{HR}_{\mathrm{NP}}$ groups revealed significant increases in activation over time in those at high risk who moved from asymptomatic to symptomatic in the left middle temporal gyrus (BA21) $\left[p_{\text {corrected }}=0.026, \mathrm{~K}_{\mathrm{E}}=112, Z=3.94(-64,-38,-6)\right.$ ], using a small volume correction for temporal lobes (Fig. 3). There were no significant differences between the $\mathrm{HR}_{\mathrm{NN}}$ and $\mathrm{HR}_{\mathrm{PN}}$ groups.

\section{Parametric contrast}

\section{Trait effects}

Formal comparison of the changes over time between the groups revealed no significant differences between the groups at the chosen levels of significance.

\section{State effects}

Comparison of the $\mathrm{HR}_{\mathrm{NN}}$ and $\mathrm{HR}_{\mathrm{NP}}$, and $\mathrm{HR}_{\mathrm{NN}}$ and $H_{P N}$ groups revealed no significant increases or decreases over time between the groups. 
(a)

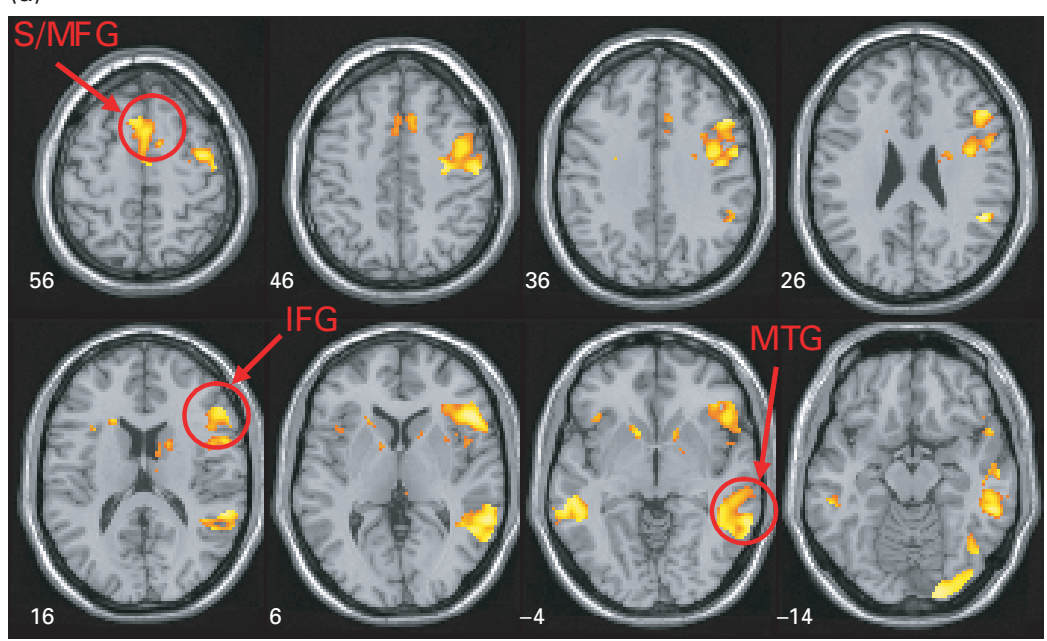

(b)
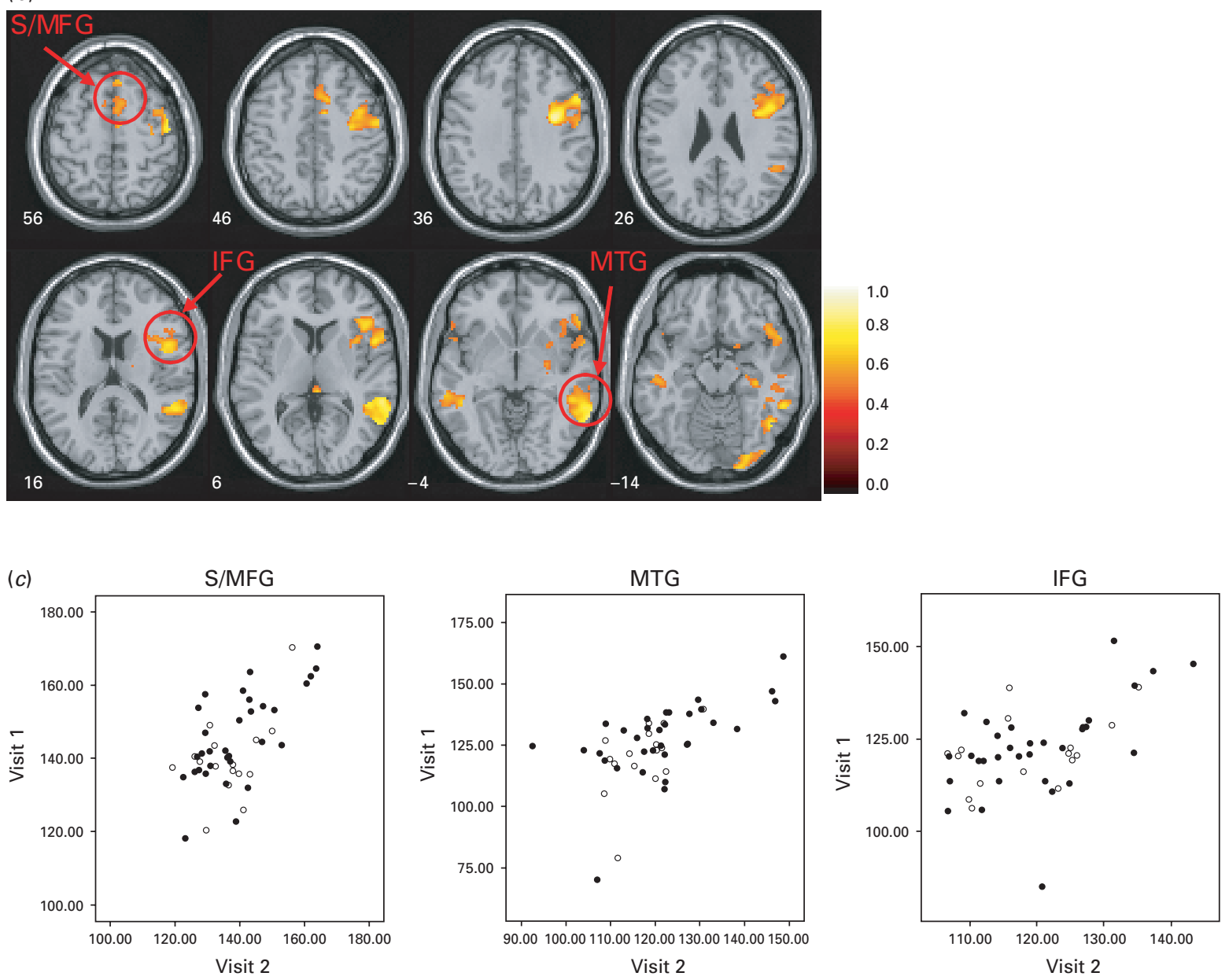

Fig. 2. Intra-class correlation coefficient values $(<0.5)$ are shown for the main regions of activation in the sentence completion versus baseline contrast for $(a)$ control subjects with no psychotic symptoms at times 1 and $2\left(\mathrm{C}_{\mathrm{NN}}\right.$ group) and (b) high-risk subjects with no psychotic symptoms at times 1 and $2\left(\mathrm{HR}_{\mathrm{NN}}\right.$ group); see Method section for further details. (c) Signal plots are presented for the three main regions of interest at visit 1 and visit 2 for the $C_{N N}(\bigcirc)$ and $H_{N N}(\bullet)$ groups. The three main regions of interest are the superior/medial frontal gyrus (S/MFG), the middle temporal gyrus (MTG) and the inferior frontal gyrus (IFG). 
Table 3. ICC values for regions of interest ${ }^{\mathrm{a}}$

\begin{tabular}{lll}
\hline Region & $\begin{array}{l}\mathrm{C}_{\mathrm{NN}} \\
(n=16)\end{array}$ & $\begin{array}{l}\mathrm{HR}_{\mathrm{NN}} \\
(n=32)\end{array}$ \\
\hline $\begin{array}{l}\text { Left superior/medial frontal } \\
\text { gyrus }(-4,4,56)\end{array}$ & 0.65 & 0.80 \\
$\begin{array}{l}\text { Left middle temporal gyrus } \\
(-54,-38,-8)\end{array}$ & 0.55 & 0.68 \\
$\begin{array}{l}\text { Left inferior frontal gyrus } \\
(-54,14,14)\end{array}$ & 0.60 & 0.65 \\
\hline
\end{tabular}

ICC, Intra-class correlation coefficient; $\mathrm{C}_{\mathrm{NN}}$, control subjects with no psychotic symptoms at times 1 and 2; $\mathrm{HR}_{\mathrm{NN}}$, high-risk subjects with no psychotic symptoms at times 1 and 2 .

a ICC values for sphere, radius $10 \mathrm{~mm}$, centred on respective coordinates.
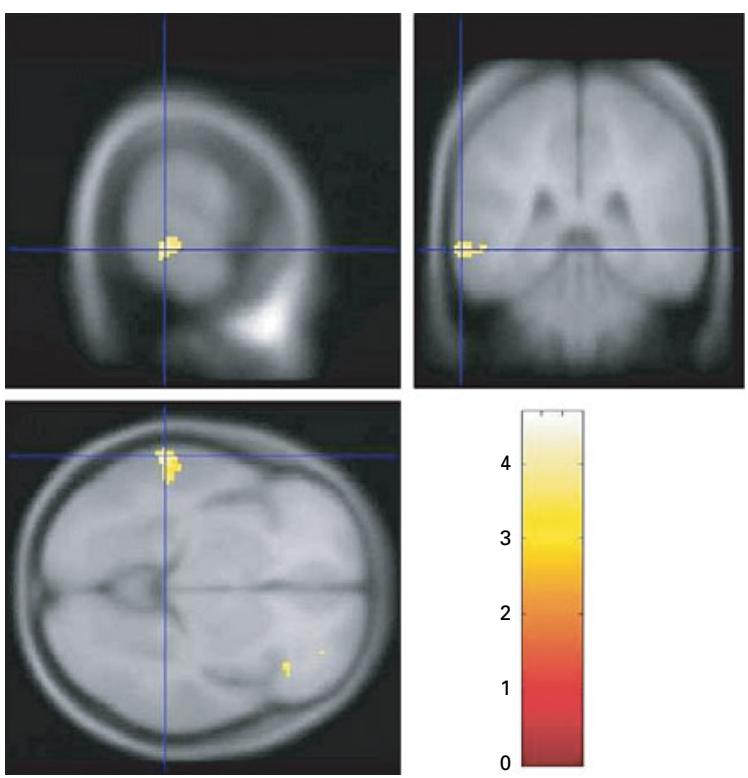

Fig. 3. State contrast: significant difference between high-risk groups in the left posterior middle temporal gyrus $(p=0.026)$, representing greater increases over time in the high-risk subjects with no psychotic symptoms at time 1 but with psychotic symptoms at time 2 ( $\mathrm{HR}_{\mathrm{NP}}$ group) versus the high-risk subjects with no psychotic symptoms at times 1 and $2\left(\mathrm{HR}_{\mathrm{NN}}\right.$ group).

\section{Discussion}

The results of this large longitudinal fMRI study conducted over a relatively long time interval (18 months) indicate no change over time between high-risk subjects and controls ('trait' effects). We did, however, see changes over time in those developing psychotic symptoms. Our results, therefore, indicate that the greatest functional changes over time in our high-risk subjects relate to changes in (sub-diagnostic) symptoms, i.e. 'state' effects. Behavioural measures indicated minimal practice effects and all those studied were naive to antipsychotic medication - hence the findings are unlikely to be attributable to such factors. Furthermore, reliability measures indicated consistent patterns of activation in the stable asymptomatic groups.

The small number of functional longitudinal studies on the established illness have in general been conducted with the purpose of examining effects of medication on changes in symptoms and brain function (Davis et al. 2005). In general, these studies suggest treatment effects or 'normalization' of brain function in frontal, temporal and cerebellar regions post-medication (Honey et al. 1999; Davis et al. 2005; Stip et al. 2005; Yurgelun-Todd et al. 2005). By design, however, these studies have been conducted over relatively short time-frames and do not address longerterm changes in function and symptoms over the course of the illness. These studies have also been accompanied by methodological limitations such as small sample sizes, lack of healthy control data, and a lack of reliability measures (Davis et al. 2005). As described earlier, the majority of reproducibility studies have been conducted on healthy control populations, and very few studies have examined reproducibility in patient populations, particularly using complex tasks (Manoach et al. 2001; Chen \& Small, 2007). Our reproducibility findings in both controls and high-risk subjects performing the current cognitive task indicating good agreement aid interpretation of the current findings and are encouraging for future longitudinal fMRI studies.

In line with our original hypothesis regarding progressive changes in the temporal lobe, fluctuating symptom effects were associated with changes in activation in the lateral temporal cortex. High-risk subjects who moved from asymptomatic to symptomatic demonstrated increasing activation in the left middle temporal gyrus. Although to our knowledge there are no other longitudinal fMRI studies in high-risk individuals, our findings are in general consistent with the available literature regarding progressive structural changes in grey matter in the lateral temporal cortex during early stages of the illness (Kasai et al. 2003a,b; Whitford et al. 2006) and in high-risk individuals (Thompson et al. 2001; Lawrie et al. 2002; Pantelis et al. 2003; Job et al. 2005). Indeed, the lateral temporal cortex has been widely implicated regarding the manifestation of psychotic symptoms in the established illness, in particular auditory hallucinations (Cleghorn et al. 1992; Suzuki et al. 1993; David et al. 1996; Dierks et al. 1999; Lennox et al. 2000; Shergill et al. 2000; Stephane et al. 2000; van de Ven et al. 2005). 
Further, although slightly more anterior to the region reported here, we have also prevously found associations between activation in the left middle temporal gyrus and the severity of hallucinations in the full baseline sample of the same high-risk cohort (Whalley et al. 2007). Due to the relatively small numbers of high-risk subjects in the asymptomatic to symptomatic group, however, we did not consider it appropriate in the current study to further split the group according to the types of symptoms experienced. It is also worth noting that the current results were seen for the contrast of sentence completion (all levels of difficulty) versus a visual baseline, rather than the more subtle and more heavily controlled parametric contrast. We cannot exclude the possibility therefore that these findings may relate to basic cognitive processes involved in the task rather than higher-level processes relating specifically to semantic memory components of the task.

One other limitation of the current study is that the group of subjects with fluctuating symptoms is relatively small. The difficulty in collecting such data, however, should not be underestimated. We also acknowledge that the finding of increasing activation when subjects move to being symptomatic would be strengthened if the reverse were seen in those moving to being asymptomatic. It is difficult to speculate why this was not the case since little is known regarding the course and fluctuation of psychotic symptoms, and indeed it may simply be the case that in clinical terms the $\mathrm{HR}_{\mathrm{NP}}$ group are not simply the 'inverse' of the $\mathrm{HR}_{\mathrm{PN}}$ group. Another methodological issue relates to the chosen group comparisons. Although a number of other comparisons were possible we considered that a restricted range of comparisons of specific groups directed at addressing our primary hypotheses was the most favourable approach in order to limit the number of comparisons made. It is also worth noting that it is not possible to say from these data whether these functional brain changes represent the cause or consequence of the symptomatic changes.

In conclusion, findings from this study indicate that fMRI activation patterns were consistent in the clinically stable groups of controls and genetic high-risk subjects. Further, they demonstrate the presence of symptom-related functional brain changes in subjects at high genetic risk of schizophrenia, indicating changes in functional activation occur in association with the development of psychotic symptoms. These findings are consistent with other literature indicating that changes in symptomatology and neurobiology are an important aspect in our improved understanding of the neural changes underpinning schizophrenia.

\section{Acknowledgements}

The study was funded by a Medical Research Council (MRC) programme grant. S.M.L. and H.C.W. are supported by a charitable donation from the Dr Mortimer and Theresa Sackler Foundation. Scanning was carried out at the BIRC for Scotland. Both BIRC and the Centre for Functional Imaging Studies (CFIS) are funded by the Scottish Higher Education Funding Council (SHEFC).

\section{Declaration of Interest}

None.

\section{References}

Aron AR, Gluck MA, Poldrack RA (2006). Long-term test-retest reliability of functional MRI in a classification learning task. Neuroimage 29, 1000-1006.

Bloom PA, Fischler I (1980). Completion norms for 329 sentence contexts. Memory and Cognition 8, 631-642.

Burgess P, Shallice T (1997). The Hayling and Brixton Tests. Thames Valley Test Company Limited: Bury St Edmunds.

Byrne M, Hodges A, Grant E, Owens DC, Johnstone EC (1999). Neuropsychological assessment of young people at high genetic risk for developing schizophrenia compared with controls: preliminary findings of the Edinburgh High Risk Study (EHRS). Psychological Medicine 29, 1161-1173.

Casey BJ, Cohen JD, O'Craven K, Davidson RJ, Irwin W, Nelson CA, Noll DC, Hu X, Lowe MJ, Rosen BR, Truwitt CL, Turski PA (1998). Reproducibility of fMRI results across four institutions using a spatial working memory task. Neuroimage 8, 249-261.

Chee MW, Lee HL, Soon CS, Westphal C, Venkatraman V (2003). Reproducibility of the word frequency effect: comparison of signal change and voxel counting. Neuroimage 18, 468-482.

Chen EE, Small SL (2007). Test-retest reliability in fMRI of language: group and task effects. Brain and Language 102, 176-185.

Cleghorn JM, Franco S, Szechtman B, Kaplan RD, Szechtman H, Brown GM, Nahmias C, Garnett ES (1992). Toward a brain map of auditory hallucinations. American Journal of Psychiatry 149, 1062-1069.

David AS, Woodruff PW, Howard R, Mellers JD, Brammer M, Bullmore E, Wright I, Andrew C, Williams SC (1996). Auditory hallucinations inhibit exogenous activation of auditory association cortex. Neuroreport 7, 932-936.

Davis CE, Jeste DV, Eyler LT (2005). Review of longitudinal functional neuroimaging studies of drug treatments in patients with schizophrenia. Schizophrenia Research 78, 45-60.

Dierks T, Linden DE, Jandl M, Formisano E, Goebel R, Lanfermann H, Singer W (1999). Activation of Heschl's gyrus during auditory hallucinations. Neuron 22, 615-621. 
Fusar-Poli P, Perez J, Broome M, Borgwardt S, Placentino A, Caverzasi E, Cortesi M, Veggiotti P, Politi P, Barale F, McGuire P (2007). Neurofunctional correlates of vulnerability to psychosis: a systematic review and meta-analysis. Neuroscience and Biobehavioral Reviews 31, 465-484.

Hafner H, Maurer K, Loffler W, Reicher-Rossler A (1993). The influence of age and sex on the onset and early course of schizophrenia. British Journal of Psychiatry 162, 80-86.

Hodges A, Byrne M, Grant E, Johnstone E (1999). People at risk of schizophrenia. Sample characteristics of the first 100 cases in the Edinburgh High-Risk Study. British Journal of Psychiatry 174, 547-553.

Honey GD, Bullmore ET, Soni W, Varatheesan M, Williams SC, Sharma T (1999). Differences in frontal cortical activation by a working memory task after substitution of risperidone for typical antipsychotic drugs in patients with schizophrenia. Proceedings of the National Academy of Sciences USA 96, 13432-13437.

Job DE, Whalley HC, Johnstone EC, Lawrie SM (2005). Grey matter changes over time in high risk subjects developing schizophrenia. Neuroimage 25, 1023-1030.

Johnstone EC, Abukmeil SS, Byrne M, Clafferty R, Grant E, Hodges A, Lawrie SM, Owens DG (2000). Edinburgh High Risk Study - findings after four years: demographic, attainment and psychopathological issues. Schizophrenia Research 46, 1-15.

Johnstone EC, Ebmeier KP, Miller P, Owens DG, Lawrie SM (2005). Predicting schizophrenia: findings from the Edinburgh High-Risk Study. British Journal of Psychiatry 186, 18-25.

Johnstone EC, Lawrie SM, Cosway R (2002). What does the Edinburgh High-Risk Study tell us about schizophrenia? American Journal of Medical Genetics 114, 906-912.

Kasai K, Shenton ME, Salisbury DF, Hirayasu Y, Lee CU, Ciszewski AA, Yurgelun-Todd D, Kikinis R, Jolesz FA, McCarley RW (2003a). Progressive decrease of left superior temporal gyrus gray matter volume in patients with first-episode schizophrenia. American Journal of Psychiatry 160, 156-164.

Kasai K, Shenton ME, Salisbury DF, Hirayasu Y, Onitsuka T, Spencer MH, Yurgelun-Todd DA, Kikinis R, Jolesz FA, McCarley RW (2003b). Progressive decrease of left Heschl gyrus and planum temporale gray matter volume in first-episode schizophrenia: a longitudinal magnetic resonance imaging study. Archives of General Psychiatry 60, 766-775.

Lawrie SM, Whalley HC, Abukmeil SS, Kestelman JN, Miller P, Best JJ, Owens DG, Johnstone EC (2002). Temporal lobe volume changes in people at high risk of schizophrenia with psychotic symptoms. British Journal of Psychiatry 181, 138-143.

Lennox BR, Park SB, Medley I, Morris PG, Jones PB (2000). The functional anatomy of auditory hallucinations in schizophrenia. Psychiatry Research 100, 13-20.

Machielsen WC, Rombouts SA, Barkhof F, Scheltens P, Witter MP (2000). fMRI of visual encoding: reproducibility of activation. Human Brain Mapping 9, 156-164.

Manoach DS, Halpern EF, Kramer TS, Chang Y, Goff DC, Rauch SL, Kennedy DN, Gollub RL (2001). Test-retest reliability of a functional MRI working memory paradigm in normal and schizophrenic subjects. American Journal of Psychiatry 158, 955-958.

Marchand WR, Lee JN, Thatcher J, Thatcher GW, Jensen C, Starr J (2007). A preliminary longitudinal fMRI study of frontal-subcortical circuits in bipolar disorder using a paced motor activation paradigm. Journal of Affective Disorders 103, 237-241.

Pantelis C, Velakoulis D, McGorry PD, Wood SJ, Suckling J, Phillips LJ, Yung AR, Bullmore ET, Brewer W, Soulsby B, Desmond P, McGuire PK (2003). Neuroanatomical abnormalities before and after onset of psychosis: a cross-sectional and longitudinal MRI comparison. Lancet 361, 281-288.

Shenton ME, Dickey CC, Frumin M, McCarley RW (2001). A review of MRI findings in schizophrenia. Schizophrenia Research 49, 1-52.

Shergill SS, Brammer MJ, Williams SC, Murray RM, McGuire PK (2000). Mapping auditory hallucinations in schizophrenia using functional magnetic resonance imaging. Archives of General Psychiatry 57, 1033-1038.

Specht K, Willmes K, Shah NJ, Jancke L (2003). Assessment of reliability in functional imaging studies. Journal of Magnetic Resonance Imaging 17, 463-471.

Stephane M, Folstein M, Matthew E, Hill TC (2000). Imaging auditory verbal hallucinations during their occurrence. Journal of Neuropsychiatry and Clinical Neurosciences 12, 286-287.

Stip E, Fahim C, Mancini-Marie A, Bentaleb LA, Mensour B, Mendrek A, Beauregard M (2005). Restoration of frontal activation during a treatment with quetiapine: an fMRI study of blunted affect in schizophrenia. Progress in Neuro-Psychopharmacology and Biological Psychiatry 29, 21-26.

Suzuki M, Yuasa S, Minabe Y, Murata M, Kurachi M (1993). Left superior temporal blood flow increases in schizophrenic and schizophreniform patients with auditory hallucination: a longitudinal case study using ${ }^{123}$ I-IMP SPECT. European Archives of Psychiatry and Clinical Neuroscience 242, 257-261.

Szaflarski JP, Schmithorst VJ, Altaye M, Byars AW, Ret J, Plante E, Holland SK (2006). A longitudinal functional magnetic resonance imaging study of language development in children 5 to 11 years old. Annals of Neurology 59, 796-807.

Thompson PM, Vidal C, Giedd JN, Gochman P, Blumenthal J, Nicolson R, Toga AW, Rapoport JL (2001). Mapping adolescent brain change reveals dynamic wave of accelerated gray matter loss in very early-onset schizophrenia. Proceedings of the National Academy of Sciences USA 98, 11650-11655.

Tombari D, Loubinoux I, Pariente J, Gerdelat A, Albucher JF, Tardy J, Cassol E, Chollet F (2004). A longitudinal fMRI study: in recovering and then in clinically stable sub-cortical stroke patients. Neuroimage 23, 827-839.

van de Ven VG, Formisano E, Roder $\mathrm{CH}$, Prvulovic $\mathrm{D}$, Bittner RA, Dietz MG, Hubl D, Dierks T, Federspiel A, Esposito F, Di Salle F, Jansma B, Goebel R, Linden DE 
(2005). The spatiotemporal pattern of auditory cortical responses during verbal hallucinations. Neuroimage 27, 644-655.

Ward NS, Brown MM, Thompson AJ, Frackowiak RS (2003). Neural correlates of motor recovery after stroke: a longitudinal fMRI study. Brain 126, 2476-2496.

Whalley HC, Gountouna VE, Hall J, McIntosh A, Whyte MC, Simonotto E, Job D, Owens DC, Johnstone EC, Lawrie SM (2007). Correlations between fMRI activation and individual psychotic symptoms in un-medicated subjects at high genetic risk of schizophrenia. BMC Psychiatry 7, 61-71.

Whalley HC, Simonotto E, Flett S, Marshall I, Ebmeier KP, Owens DG, Goddard NH, Johnstone EC, Lawrie SM (2004). fMRI correlates of state and trait effects in subjects at genetically enhanced risk of schizophrenia. Brain 127, 478-490.

Whitford TJ, Grieve SM, Farrow TF, Gomes L, Brennan J, Harris AW, Gordon E, Williams LM (2006). Progressive grey matter atrophy over the first $2-3$ years of illness in first-episode schizophrenia: a tensor-based morphometry study. Neuroimage 32, 511-519.

Wing JK, Cooper JE, Sartorius N (1974). The Description and Classification of Psychiatric Symptoms. An Instruction Manual for the PSE and Catego Systems. Cambridge University Press: Cambridge.

WHO (1993). ICD-10: The ICD-10 Classification of Mental and Behavioural Disorders : Diagnostic Criteria for Research. World Health Organization: Geneva.

Yoo SS, O'Leary HM, Dickey CC, Wei XC, Guttmann CR, Park HW, Panych LP (2005). Functional asymmetry in human primary auditory cortex: identified from longitudinal fMRI study. Neuroscience Letters 383, 1-6. Yurgelun-Todd DA, Coyle JT, Gruber SA, Renshaw PF, Silveri MM, Amico E, Cohen B, Goff DC (2005). Functional magnetic resonance imaging studies of schizophrenic patients during word production: effects of D-cycloserine. Psychiatry Research 138, 23-31. 